November 4, 2018

\title{
NON-SINGULAR SOLUTIONS TO THE NORMALIZED RICCI FLOW EQUATION
}

\author{
FUQUAN FANG, YUGUANG ZHANG, AND ZHENLEI ZHANG
}

\begin{abstract}
In this paper we study non-singular solutions of Ricci flow on a closed manifold of dimension at least 4. Amongst others we prove that, if $M$ is a closed 4-manifold on which the normalized Ricci flow exists for all time $t>0$ with uniformly bounded sectional curvature, then the Euler characteristic $\chi(M) \geq 0$. Moreover, the 4-manifold satisfies one of the following

(i) $M$ is a shrinking Ricci solition;

(ii) $M$ admits a positive rank $F$-structure;

(iii) the Hitchin-Thorpe type inequality holds$$
2 \chi(M) \geq 3|\tau(M)|
$$

where $\chi(M)$ (resp. $\tau(M))$ is the Euler characteristic (resp. signature) of $M$.
\end{abstract}

\section{INTRODUCTION}

In this paper we will consider the normalized Ricci flow equation on a closed smooth $n$-dimensional manifold $M$,

$$
\left\{\begin{array}{l}
\frac{\partial}{\partial t} g=-2 R i c+\frac{2 r}{n} g \\
g(0)=g_{0},
\end{array}\right.
$$

where Ric and $r$ denote the Ricci tensor and average scalar curvature $\frac{\int_{M} R d v}{\int_{M} d v}$ of the Riemannian metric $g$ respectively. Following Hamilton [12, a solution to this equation is called non-singular, if the flow exists for all time $t \geq 0$ and the Riemannian curvature tensor satisfies $|R m| \leq C<\infty$ uniformly for some constant $C$ independent of $t$.

In 12, Hamilton classified 3-dimensional non-singular solutions. In particular, he proved that the underlying 3-manifold is geometrizable in the sense of Thurston. Hamilton's theorem is of great importance to understand the long-time behavior of solutions to the Ricci flow, even to the Ricci flow with surgery which was used by Perelman in 17] as a technique to study the global property of the Ricci flow solution, modulo modifying the singular points in space time (cf. for example [7] or [14] for detailed discussion.)

Our main result is the following generalization of Hamilton's results to higher dimensions.

Theorem 1.1. Any non-singular solution to the normalized Ricci flow equation (1) on a closed manifold $M$ does one and only one of the following things:

\section{(1.1.1) the solution collapses;}

The first author was supported by NSF Grant 19925104 of China, 973 project of Foundation Science of China, and the Capital Normal University. 
(1.1.2) the solution converges along a subsequence to a shrinking Ricci soliton solution on $M$;

(1.1.3) the solution converges along a subsequence to a Ricci flat metric solution on $M$;

(1.1.4) the solution converges along a subsequence to a negative Einstein metric solution on $M$;

(1.1.5) the solution converges along a subsequence of times and space points to a complete non-compact negative Einstein metric solution in the pointed Gromov-Hausdoff sense.

A solution $(M, g(t))$ to equation (11) is called collapse if the maximum of the local injectivity radius $\operatorname{inj}(x, g(t)), x \in M$, tends to zero along some subsequence of times $t_{k} \rightarrow \infty$.

In dimension 3, by a result of Ivey [13, every closed Ricci soliton is Einstein. So the convergence result coincides with that of Hamilton's. For case (1.1.5), Hamilton proved that the 3-manifold splits into pieces of hyperbolic manifolds and residual graph manifolds. Each hyperbolic piece has finite volume and finite cusps at infinity, every cusp contains an incompressible torus $T$, i.e., $\pi_{1}(T)$ injects into $\pi_{1}(M)$, with constant mean curvature and small area. In general, by Cheeger-Gromov [3], the Riemannian manifold $(M, g(t))$ for large $t$ admits a thick-thin decomposition $M=M^{\varepsilon} \cup M_{\varepsilon}$ for small $\varepsilon$, where

$$
\begin{aligned}
& M^{\varepsilon}=\{x \in M \mid \operatorname{Vol}(B(x, 1, g(t))) \geq \varepsilon\}, \\
& M_{\varepsilon}=\{x \in M \mid \operatorname{Vol}(B(x, 1, g(t))) \leq \varepsilon\} .
\end{aligned}
$$

By (1.1.5) it is easy to see that the thick part is recognized as the negative Einstein pieces, while the thin part has an $F$-structure which partially collapses when we take the limit (cf. [1, Thm. 1.3]). In 4-dimension we will prove the thin part is indeed volume collapsed (cf. Theorem 1.5).

The following is a generalization of the celebrated Hitchin-Thorpe inequality for nonsingular solutions to the Ricci flow on closed 4-manifolds.

Theorem 1.2. Let $M$ be a closed oriented 4-manifold $M$, and $\{g(t)\}, t \in[0, \infty)$, be a non-singular solution to (11). Then $M$ satisfies one of the following

(1.2.1) $M$ admits a shrinking Ricci solition;

(1.2.2) $M$ admits a positive rank F-structure;

(1.2.3) the Hitchin-Thorpe type inequality holds

$$
2 \chi(M) \geq 3|\tau(M)|
$$

where $\chi(M)$ (resp. $\tau(M)$ ) is the Euler characteristic (resp. signature) of $M$.

By [8] a closed shrinking Ricci soliton has finite fundamental group. Thus, a 4dimensional closed shrinking Ricci soliton has Euler characteristic at least 2. On the other hand, manifold with positive rank $F$-structure has vanishing Euler characteristic. Therefore Theorem 1.2 implies readily that

Corollary 1.3. If a closed 4-manifold $M$ admits a non-singular solution, then $\chi(M) \geq$ 0 . 
The above corollary gives a topological obstruction to the existence of non-singular solutions on a closed 4-manifold.

To state a strengthened result of Theorem 1.2 we need to introduce Perelman's $\lambda$ functional (cf. [17] 14] 8]).

For a smooth function $f \in C^{\infty}(M)$ on a Riemannian $n$-manifold with a Riemannian metric $g$, let

$$
\mathcal{F}(g, f)=\int_{M}\left(R_{g}+|\nabla f|^{2}\right) e^{-f} d v o l_{g}
$$

where $R_{g}$ is the scalar curvature of $g$. The Perelman's $\lambda$-functional is defined by

$$
\lambda_{M}(g)=\inf _{f}\left\{\mathcal{F}(g, f) \mid \int_{M} e^{-f} d v l_{g}=1\right\} .
$$

Note that $\lambda_{M}(g)$ is the lowest eigenvalue of the operator $-4 \triangle+R_{g}$. Let

$$
\bar{\lambda}_{M}(g)=\lambda_{M}(g) \operatorname{Vol}_{g}(M)^{\frac{2}{n}}
$$

which is invariant up to rescale the metric. Perelman [17] has established the monotonicity property of $\bar{\lambda}_{M}\left(g_{t}\right)$ along the Ricci flow $g_{t}$, namely, the function is non-decreasing along the Ricci flow $g_{t}$ whenever $\bar{\lambda}_{M}\left(g_{t}\right) \leq 0$. Therefore, it is interesting to study the upper bound of $\bar{\lambda}_{M}(g)$. This leads to define a diffeomorphism invariant $\bar{\lambda}_{M}$ of $M$ due to Perelman (cf. [18] [14) by

$$
\bar{\lambda}_{M}=\sup _{g \in \mathcal{M}} \bar{\lambda}_{M}(g)
$$

where $\mathcal{M}$ is the set of Riemannian metrics on $M$. As we pointed out in 8$] \bar{\lambda}_{M}$ is not a homeomorphism invariant. Observe that $\bar{\lambda}_{M}=0$ if $M$ admits a volume collapsing with bounded scalar curvature but does not admit any metric with positive scalar curvature (cf. [14]).

A upper bound in 8 for the invariant $\bar{\lambda}_{M}$ was obtained by using the Seiberg-Witten theory, whenever $\bar{\lambda}_{M}<0$ and $M$ has a non-trivial monopole class.

Now we are ready to state

Theorem 1.4. Let $M$ be a closed oriented 4-manifold $M$ with $\bar{\lambda}_{M}<0$, and $\{g(t)\}, t \in$ $[0, \infty)$, be a solution to (11). If $|R(g(t))|<C$ where $C$ is a constant independent of $t$, then

$$
2 \chi(M)-3|\tau(M)| \geq \frac{1}{96 \pi^{2}} \bar{\lambda}_{M}^{2},
$$

In particular, if $\{g(t)\}, t \in[0, \infty)$ is a non-singular solution to (11), then the solution $(M, g(t))$ does not collapse.

The above theorem combined with the Seiberg-Witten theory implies that

Corollary 1.5. Let $M$ be a closed symplectic 4-manifold $M$ with $\bar{\lambda}_{M}<0$, and $\{g(t)\}, t \in$ $[0, \infty)$, be a solution to (1) with $|R(g(t))|<C$ where $C$ is a constant independent of $t$. 
If $b_{2}^{+}(M):=\operatorname{dim}_{+}^{2}(M ; \mathbb{R})>1$, then

$$
\chi(M) \geq 3 \tau(M) .
$$

In dimension 4 Theorem (1.1.5) may be improved as follows:

Theorem 1.6. Let $(M, g(t)), t \in[0, \infty)$ be a non-singular solution to (1) on a closed oriented 4-manifold $M$ with $\bar{\lambda}_{M}<0$. Then, for any $\delta>0$, there is a time $T \gg 1$, and a compact 4-submanifold $M^{\varepsilon}$ with boundary in $M, M^{\varepsilon} \subset M$, such that

(1.5.1) $\operatorname{Vol}\left(M-M^{\varepsilon}, g(T)\right)<\delta$, and $M-M^{\varepsilon}$ admits an F-structure of positive rank.

(1.5.2) The components of $\partial M^{\varepsilon}$ are graph 3-manifolds.

(1.5.3) $M^{\varepsilon}$ admits an Einstein metric with negative scalar curvature $g_{\infty}$ which is close to $\left.g(T)\right|_{M^{\varepsilon}}$ in the $C^{\infty}$-sense.

In view of Theorem 1.6, it is natural to wonder what kind of information can be obtained on the thick part $M^{\varepsilon}$ in the above theorem. Applying the Seiberg-Witten theory we obtain the following result which provides a partial answer for certain symplectic manifolds.

Theorem 1.7. Let $(M, g(t))$ and $\left(M^{\varepsilon}, g_{\infty}\right)$ be the same as in Theorem 1.6. If $M$ admits a symplectic structure satisfying that $b_{2}^{+}(M)>1$ and $\chi(M)=3 \tau(M)$, then $g_{\infty}$ is a complex hyperbolic metric.

We conclude this section by posing the following

Conjecture 1.8. Theorem (1.2.3) may be replaced by the following Hitchin-ThorpeGromov-Kotschik type inequality

$$
2 \chi(M)-3|\tau(M)| \geq \frac{1}{1296 \pi^{2}}\|M\|
$$

where $\|M\|$ is a simplicial volume of $M$.

The organization of the paper is as follows: In $\S 2$ we give a proof of Theorem 1.1. In $\S 3$ we are concerned with 4-dimensional non-singular solutions, and we will prove Theorem 1.2, Theorem 1.4 and Corollary 1.5. In $\S 4$ we will prove Theorem 1.6. Finally, we will prove Theorem 1.7 in $\S 5$.

\section{Proof of Theorem 1.1}

In [10, Hamilton introduced the (unnormalized) Ricci flow equation

$$
\left\{\begin{array}{l}
\frac{\partial}{\partial t} g=-2 R i c \\
g(0)=g_{0}
\end{array}\right.
$$

and its normalized equation (11). By [10] the equation (11) is just a change of equation (6) via rescalings in space and a reparametrization in time, such that the volumes of the Riemannian metrics are preserved to be constant. In this paper, we will always assume 
the volumes of the metrics equal 1 , whenever we consider the solutions to the normalized Ricci flow. Especially we have $\operatorname{Vol}\left(g_{0}\right)=1$.

As in [17, one also can define a scale invariant version of $\mathcal{F}$ functional, the so called $\mathcal{W}$ functional. For each smooth $f$ and constant $\tau>0$, let

$$
\mathcal{W}(g, f, \tau)=\int_{M}\left[\tau\left(R+|\nabla f|^{2}\right)+f-n\right](4 \pi \tau)^{-n / 2} e^{-f} d v
$$

and then set

$$
\begin{gathered}
\mu(g, \tau)=\inf \left\{\mathcal{W}(g, f, \tau) \mid \int_{M}(4 \pi \tau)^{-n / 2} e^{-f} d v=1\right\}, \\
\nu(g)=\inf _{\tau>0} \mu(g, \tau) .
\end{gathered}
$$

By a result of Rothaus [19], for each $\tau>0$, there exists a smooth minimizer $\phi$ such that $\mu(g, \tau)=\mathcal{W}(g, \phi, \tau)$. By Claim 3.1 of [17], if $\lambda(g)>0$, then the infimum in the definition of $\nu$ is attained by some $\tau>0$ and $\nu(g) \leq 0$. By [17 the $\lambda$ functional and the $\nu$ functional are non-decreasing along the Ricci flow, which plays a central role in our proof (cf. Lemma 2.11 and Lemma 2.12 for proofs of these facts.)

We start with the definition of convergence of solutions to the equation (11) or (6). We assume that all the Riemannian metrics taking into account are complete.

Definition 2.1 ([1] $)$. Let $\left(M_{k}, g_{k}(t), p_{k}\right), p_{k} \in M, t \in(A, B)$ with $-\infty \leq A<0$ and $0<B \leq \infty$, be a sequence of marked solutions to the Ricci flow equation (11) or (6). We say that $\left(M_{k}, g_{k}(t), p_{k}\right)$ converges to another solution $\left(M_{\infty}, g_{\infty}(t), p_{\infty}\right), t \in(A, B)$, to (11) or (6) respectively, if there is a sequence of increasing open subsets $U_{k} \subset M_{\infty}$ containing $p_{\infty}$, i.e., $U_{k} \subset U_{k+1}$ for each $k$, and a sequence of diffeomorphisms $F_{k}: U_{k} \rightarrow V_{k} \subset M_{k}$ mapping $p_{\infty}$ to $p_{k}$, such that the pull-backed metrics $F_{k}^{*} g_{k}(t)$ converge to $g_{\infty}(t)$ on every compact subset of $M_{\infty} \times(A, B)$ uniformly together with all their derivatives.

In [1], Hamilton proved his famous compactness theorem for solutions to the unnormalized Ricci flow equation ([6), under the assumption: (i) the local injectivity radii of the metrics $g_{k}(0)$ at $p_{k}$ are uniformly bounded below; (ii) the supremum norm of Riemannian curvature tensors are uniformly bounded above on any compact time interval. For a local version of this theorem, see [7] for example. We remark that the compactness theorem of Hamilton remains valid for the normalized Ricci flow solutions under the same assumption. This can be checked easily from the proof of the theorem.

Recall that $(M, g(t))$ is a Ricci solition solution to the Ricci flow, if $g(t)$ is obtained from $g(0)$ via changes by diffeomorphisms and rescalings. At each time the solution satisfies

$$
R i c+\mathcal{L}_{X} g=c g
$$

for some vector field $X$ and some constant $c$. The Ricci soliton is said to be shrinking, steady or expanding according to $c>0, c=0, c<0$ respectively. Note that if the manifold is closed, then $c=r$, which can be seen by taking the trace of above equation and then integrating it over $M$, where $r$ is the average scalar curvature of $g$. 
In [12, Hamilton considered metrics with positive scalar curvature. Our next proposition deals with the solutions so that the $\lambda$-functional is positive.

Proposition 2.2. Let $(M, g(t)), t \in[0, \infty)$, be a non-singular solution to (1) on a closed $n$-manifold $M$ with $\lambda(g(0))>0$. Then for any sequence of times $t_{k} \rightarrow \infty$, there is a subsequence $t_{k_{i}}$ such that $\left(M, g\left(t_{k_{i}}+t\right)\right)$ converges to a shrinking Ricci soliton solution.

Proof. If we denote by $(M, \bar{g}(t))$ the corresponding unnormalized Ricci flow solution, then by Proposition 1.2 of [17,

$$
\frac{d}{d t} \lambda(\bar{g}(t)) \geq \frac{2}{n} \lambda(\bar{g}(t))^{2}
$$

Thus the unnormalized Ricci flow must extinct in finite time and $\lambda(\bar{g}(t))>0$ remains hold. Since $g(t)$ are just rescalings of $\bar{g}(t), \lambda(g(t))>0$ is preserved. Then Perelman's no local collapsing theorem [17, Chap. 4] tells us that there exist $\kappa, \rho>0$ such that each metric ball $B=B_{g(t)}(p, r) \subset M$, with radius $r \leq \rho$ and $\sup _{x \in B}|R m|(x, t) \leq r^{-2}$, has volume $\operatorname{Vol}_{g(t)}(B) \geq \kappa r^{n}$. By the definition of non-singular solution, there is a constant $C>0$ such that $|R m|(x, t) \leq C$ for all $(x, t) \in M \times[0, \infty)$. By replacing $\rho$ by a smaller constant, we may assume $\rho \leq C^{-\frac{1}{2}}$. Thus $|R m|(x, t) \leq \rho^{-2}$ always hold and so $\operatorname{Vol}\left(B_{g(t)}(x, \rho)\right) \geq \kappa \rho^{n}$ for all $(x, t)$. This implies that the diameters of $g(t)$ are bounded above uniformly, since we can fill only $\frac{1}{\kappa \rho^{n}}$ disjoint balls of radius $\rho$ in $M$ at each time $t$. By a result of Cheeger, Gromov and Taylor [6], the injectivity radii of $g(t)$ are bounded below uniformly for any $t$. Then Hamilton's compactness theorem yields the convergence result.

Let $\left(M, g_{k}(t)=g\left(t_{k}+t\right)\right), t_{k} \rightarrow \infty$, be such a sequence which converges to a limit solution to the normalized Ricci flow equation $\left(M_{\infty}, g_{\infty}(t)\right), t \in(-\infty, \infty)$. By the uniform boundedness of diameters of $g(t), M_{\infty}=M$ and the convergence is smooth on any compact time interval. Next we will show that $\left(M, g_{\infty}(t)\right)$ is a shrinking Ricci soliton.

Now for each time $t \in(-\infty, \infty), \nu(g(t))$ is achieved by some positive number $\tau(t)$, i.e., $\nu(g(t))=\mu(g(t), \tau(t)) \leq 0$ holds. Further, by Lemma 2.12 below, $\nu(g(t))$ increases. By the smooth convergence of $\left(M, g_{k}(t)\right)$ for any $t$, we have

$$
\nu\left(g_{\infty}(t)\right)=\lim _{k \rightarrow \infty} \nu\left(g\left(t_{k}+t\right)\right)=\lim _{k \rightarrow \infty} \nu\left(t_{k}+t\right)=\lim _{t \rightarrow \infty} \nu(t),
$$

is a constant independent of $t$. We consider two subcases for the limit solution.

Case 1: $\lambda\left(g_{\infty}\left(t_{0}\right)\right)>0$ for some $t_{0} \geq 0$. In this case, $\nu\left(g_{\infty}(t)\right)$ is attainable for $t$ around $t_{0}$ and using Lemma 2.12 again, one sees that $\left(M, g_{\infty}(t)\right)$ is really a Ricci soliton. By definition of $\lambda$, the average scalar curvature $r\left(g_{\infty}\left(t_{0}\right)\right) \geq \lambda\left(g_{\infty}\left(t_{0}\right)\right)>0$, so the Ricci soliton is a shrinking one.

Case 2: $\lambda\left(g_{\infty}(t)\right) \equiv 0$. In this case, the monotonicity of the $\lambda$ functional implies that $\left(M, g_{\infty}(t)\right)$ is Einstein. See Lemma 2.11 below. The scalar curvature is zero because it equals $\lambda\left(g_{\infty}(t)\right)$. We will exclude this exception by showing that $\nu\left(g_{\infty}(t)\right)=-\infty$, which is a contradiction since $\nu\left(g_{\infty}(t)\right)=\lim _{t \rightarrow \infty} \nu(g(t)) \geq \nu(g(0))$ by the increasing of $\nu(g(t))$ along the Ricci flow. Hence the proposition from the following claim. 
Claim 2.3. Let $(N, g)$ be a closed Riemannian manifold with average scalar curvature $r \leq 0$. Then we have $\lim _{\tau \rightarrow \infty} \mu(g, \tau)=-\infty$.

Proof of the claim. For any smooth function $f$, set $u=(4 \pi \tau)^{-n / 2} e^{-f}$; then by the definition of the $\mathcal{W}$ functional,

$$
\mathcal{W}(g, f, \tau)=\int_{M}\left[\tau\left(R u+\frac{|\nabla u|^{2}}{u}\right)-u \ln u\right] d v-\frac{n}{2} \ln (4 \pi \tau)-n .
$$

Choosing $u=1$ and substituting it into the functional, we have

$$
\mu(g, \tau)+n \leq \tau r-\frac{n}{2} \ln (4 \pi \tau) \leq-\frac{n}{2} \ln (4 \pi \tau) \rightarrow-\infty
$$

as $\tau \rightarrow \infty$. This ends the proof of the claim.

From the proof of above proposition, we have an immediate corollary.

Corollary 2.4. If $(M, g)$ is a closed Riemannian manifold with $\lambda(g)>0$, then the normalized Ricci flow solution, with $g$ as initial metric, will never converge to a Ricci flat metric.

By a result of Fernández-López and García-Río 9], any shrinking Ricci soliton has finite fundamental group, so we have

Corollary 2.5. Let $(M, g)$ be a closed Riemannian manifold with $\lambda(g)>0$. If the normalized Ricci flow solution on $M$ with $g$ as initial metric is non-singular, then $\pi_{1}(M)$ is finite.

For a Riemannian metric $g$ on a manifold $M$, denote by $\operatorname{inj}(x, g)$ the injectivity radius of the metric $g$ at $x$. We say a solution to the normalized Ricci flow collapses if there exists a sequence of times $t_{k} \rightarrow T$ such that $\sup _{x \in M} \operatorname{inj}\left(x, g\left(t_{k}\right)\right) \rightarrow 0$, where $T$ is the maximal existence time for the solution, which may be finite or infinite. If a non-singular solution to the normalized Ricc flow equation doesn't collapse, then by Hamilton's compactness theorem, for each sequence of times $t_{k} \rightarrow \infty$, there exists a subsequence $t_{k_{i}}$ and a sequence of points $p_{i} \in M$ such that $\left(M, g\left(t_{k_{i}}+t\right), p_{i}\right)$ converges to another normalized Ricci flow solution $\left(M_{\infty}, g_{\infty}(t), p_{\infty}\right)$. Denote by $r_{\infty}(t)=\lim _{i \rightarrow \infty} r\left(g\left(t_{k_{i}}+t\right)\right)$ the limit constant in the normalized Ricci flow equation, then we have

$$
\frac{\partial}{\partial t} g_{\infty}(t)=-2 R i c_{\infty}(t)+\frac{2}{n} r_{\infty}(t) g_{\infty}(t),
$$

where $R i c_{\infty}(t)$ is the Ricci tensor of $g_{\infty}(t)$. Note that $r_{\infty}(t)$ may not equal to the average scalar curvature $r\left(g_{\infty}(t)\right)$.

Denote by $\breve{R}(g)=\min _{x \in M} R(x)$ the minimum of the scalar curvature of a given metric $g$.

Proposition 2.6. Let $(M, g(t)), t \in[0, \infty)$, be a non-singular solution to (11) on a closed $n$-manifold $M$. Assume that $\breve{R}(g(t)) \leq-c<0$ uniformly for some constant $c>0$ independent of $t$. If the solution doesn't collapse, then there exists a sequence of points 
$p_{k} \in M$ and a sequence of times $t_{k} \rightarrow \infty$ such that the solutions $\left\{\left(M, g\left(t_{k}+t\right), p_{k}\right)\right\}_{k=1}^{\infty}$ converge to an Einstein metric solution to (1), whose scalar curvature is negative.

For proving this proposition, we need the following lemma:

Lemma 2.7. Let $(M, g(t)), t \in[0, \infty)$ be a solution to (1) on a closed $n$-manifold $M$. Assume that $\breve{R}(g(t)) \leq-c<0$ uniformly for some constant $c>0$ independent of $t$. If $|R(g(t))| \leq C$, where $C$ is a constant independent of $t$, then

$$
\begin{gathered}
\int_{0}^{\infty}(r(g(t))-\breve{R}(g(t))) d t<\infty, \quad \text { and } \\
\int_{0}^{\infty} \int_{M}|R(g(t))-r(g(t))| d v d t<\infty .
\end{gathered}
$$

Proof. We follow the proof given in Section 7 of [12 by Hamilton. Consider the evolution equation of $R$

$$
\frac{\partial}{\partial t} R=\triangle R+2\left|R i c^{\circ}\right|^{2}+\frac{2}{n} R(R-r),
$$

where $R i c^{\text {o }}$ denotes the traceless part of Ricci tensor. By maximal principle, $\frac{d}{d t} \breve{R} \geq$ $\frac{2}{n} \breve{R}(\breve{R}-r)$ and so $\breve{R}(g(t))$ increases whenever it is negative. By assumption $\frac{d}{d t} \breve{R} \geq$ $\frac{2}{n} \breve{R}(\breve{R}-r) \geq \frac{2 c}{n}(r-\breve{R})$, which implies that

$$
\int_{0}^{\infty}(r-\breve{R}) d t<\infty
$$

Come back to the original solution $(M, g(t))$. We have

$$
\int_{M}|R-r| d v \leq \int_{M}(R-\breve{R}) d v+\int_{M}(r-\breve{R}) d v=2 \int_{M}(r-\breve{R}) d v=2(r-\breve{R}) .
$$

Thus

$$
\int_{0}^{\infty} \int_{M}|R-r| d v d t<\infty
$$

Proof of Proposition [2.6. Assume that $\lim _{t \rightarrow \infty} \breve{R}(t)=-\delta \leq-c<0$. Note that by assumption, there exists a constant $C>0$ such that $|R m|(x, t) \leq C$ uniformly for all $(x, t) \in M \times[0, \infty)$. First we will show that $\lim _{t \rightarrow \infty} r(t)$ exists and equals to $-\delta$. For this, consider the evolution equation

$$
\frac{d}{d t}(r-\breve{R}) \leq \int_{M}\left(2|R i c|^{2}-\frac{2}{n} R r\right) d v-\frac{2}{n} \breve{R}(\breve{R}-r) \leq D,
$$

where $D \geq 1$ is a constant depending only on $C$ and $n$. We claim that for any $0<\epsilon<1$, there is $T$ such that $r(t)-\breve{R}(t)<\epsilon$ whenever $t>T$. Otherwise, there will be a sequence 
of times $t_{k} \rightarrow \infty$ satisfying $t_{k+1} \geq t_{k}+1$ and $r\left(t_{k}\right)-\breve{R}\left(t_{k}\right) \geq \epsilon$. From the above equation, we obtain that $r(t)-\breve{R}(t) \geq \frac{\epsilon}{2}$ whenever $t \in\left[t_{k}-\epsilon / D, t_{k}\right]$. Thus

$$
\int_{0}^{\infty}(r-\breve{R}) d t \geq \sum_{k=1}^{\infty} \int_{t_{k}-\epsilon / D}^{t_{k}}(r-\breve{R}(t)) d t \geq \sum_{k=1}^{\infty} \int_{t_{k}-\epsilon / D}^{t_{k}} \frac{\epsilon}{2} d t=\infty
$$

which contradicts the Lemma2.7 Hence $\lim _{t \rightarrow \infty} r(t)=-\delta$ and consequently $r_{\infty}(t)=-\delta$ on any limit solution as we mentioned in the paragraphs before Proposition 2.6.

Considering the time interval $\left[t_{0}, t_{0}+1\right], \forall t_{0} \in \mathbb{R}$, on the limit solution, we have $\int_{t_{0}}^{t_{0}+1} \int_{M_{\infty}}\left|R\left(g_{\infty}(t)\right)-r_{\infty}(t)\right| d v d t=0$. Hence $R\left(g_{\infty}(t)\right) \equiv r_{\infty}(t)=-\delta$. Then by the evolution of scalar curvature we have $\operatorname{Ric}^{\mathrm{o}}\left(g_{\infty}(t)\right)=0$, i.e., $\left(M_{\infty}, g_{\infty}(t)\right)$ is Einstein for all $t$. The scalar curvature $R_{\infty}(t)=\lim _{t \rightarrow \infty} \breve{R}(t)=-\delta<0$.

Remark 2.8. It is remarkable that the conclusion in Proposition 2.6] remains valid if we replace the boundedness of the Riemannian curvature tensor by the boundedness of the Ricci tensor. This can be seen from the process of proving the Proposition [2.6. Also note that in both of these special cases, the limit constant $r_{\infty}(t)$ equal to $r\left(g_{\infty}(t)\right)$, the average scalar curvature of the limit metrics.

By now for a solution to the normalized Ricci flow equation, if $\lambda(g(t))>0$ for some time $t$, then we can use Proposition 2.2] while if $\breve{R}(g(t)) \leq-c<0$ holds for all $t$, then we can use Proposition 2.6. As for the remaining case, we have the following proposition similar as the zero sectional curvature limit case considered by Hamilton [12].

Proposition 2.9. Let $(M, g(t)), t \in[0, \infty)$, be a non-singular solution to (1) on a closed $n$-manifold $M$. Assume that $\breve{R}(g(t)) \leq 0, \lambda(g(t)) \leq 0$ and $\breve{R}(g(t)) \nearrow 0$ as $t \rightarrow \infty$. If the solution doesn't collapse, then there exists a sequence of times $t_{k} \rightarrow \infty$ such that the solutions $\left\{\left(M, g\left(t_{k}+t\right)\right)\right\}_{k=1}^{\infty}$ converge to a Ricci flat metric solution to (11).

Proof. We divide the proof into several subcases.

Case 1: The unnormalized Ricci flow extincts in finite time. Perelman's no local collapsing theorem shows that the limit manifold is closed and so $M_{\infty}=M$. Now $\lambda(g(t)) \nearrow 0$ implies that $\lambda\left(g_{\infty}(t)\right)=0$ for all $t$. By Lemma 2.11. $\left(M, g_{\infty}(t)\right)$ is a Ricci flat solution.

Case 2: The unnormalized Ricci flow exists for all time $t \in[0, \infty)$ and there is a sequence of times $t_{k} \rightarrow \infty$ such that the average scalar curvature $r\left(t_{k}\right)$ of $g\left(t_{k}\right)$ converges to zero. In this case, we use a modified version of the proof used in Section 6 of [12] by Hamilton. Denote by $R_{\infty}$ and $R i c_{\infty}$ the scalar curvature and Ricci tensor of the limit solution respectively. By assumption,

$$
\int_{M}\left(R\left(t_{k}\right)-\breve{R}\left(t_{k}\right)\right) d v_{g\left(t_{k}\right)}=r\left(t_{k}\right)-\breve{R}\left(t_{k}\right) \rightarrow 0
$$

as $k \rightarrow \infty$. Note that $R\left(t_{k}\right)-\breve{R}\left(t_{k}\right) \geq 0$. By taking the limit, one obtains $\int_{M_{\infty}} R_{\infty}(0) d v_{g_{\infty}}(0)=$ 0 . But $R_{\infty}(0) \geq 0$ over $M_{\infty}$ since $\lim _{k \rightarrow \infty} \breve{R}\left(t_{k}\right)=0$, so $R_{\infty}(0) \equiv 0$. Note that $r_{\infty}(0)=0$, 
since $r\left(t_{k}\right) \rightarrow 0$. Consider the evolving equation of the scalar curvature on the limit solution

$$
\frac{\partial}{\partial t} R_{\infty}(t)=\triangle R_{\infty}(t)+2\left|R i c_{\infty}(t)\right|^{2}-\frac{2}{n} r_{\infty}(t) R_{\infty}(t), t \in(-\infty, \infty) .
$$

It follows from the strong maximal principle that $R_{\infty} \equiv 0$ and $R i c_{\infty} \equiv 0$ over $M_{\infty} \times$ $(-\infty, \infty)$, i.e., $\left(M_{\infty}, g_{\infty}(t)\right)$ is a Ricci flat solution. Now the volume of the limit manifold is less than or equals to 1 . By a result of Yau [21], $M_{\infty}$ is compact. So $M_{\infty}=M$ and the convergence is smooth.

Case 3: The unnormalized Ricci flow, say $(M, \bar{g}(t))$, exists for all time $t \in[0, \infty)$ and the average scalar curvature of normalized Ricci flow $r(t) \geq \delta$ uniformly for some constant $\delta>0$. We want to show this case will never happen. Denote by $\bar{V}(t)=$ $\operatorname{Vol}(\bar{g}(t))$ the volume of $\bar{g}(t)$. Since $r(g) \operatorname{Vol}(g)^{\frac{2}{n}}$ is scale invariant, we have $\bar{r}(t) \geq \delta \bar{V}^{\frac{-2}{n}}(t)$. By the evolving equation

$$
\frac{d}{d t} \bar{V}=\int_{M}-R(\bar{g}) d v_{\bar{g}}=-\bar{r} \bar{V} \leq-\delta \bar{V}^{\frac{n-2}{n}}
$$

we obtain $\bar{V}^{\frac{2}{n}}(t) \leq 1-\frac{2}{n} \delta t$ for all $t$, which contradicts with the assumption that the unnormalized Ricci flow solution exists for all time. The desired result follows.

Remark 2.10. In fact, by a refined argument using the monotonicity of Perelman's $\mu$ functional along the Ricci flow, Case 1 of Proposition 2.9] also can be excluded. So the only phenomena is that of Case 2 , under the assumption in the proposition. 1.1.

Summing up the results of Proposition 2.2, 2.6 and 2.9, we finish the proof of Theorem

To conclude this section, let us prove two lemmas used previously, which are basically due to Perelman. These are basic facts in the study of Ricci flow solutions.

Lemma 2.11 (Perelman). Let $(M, g(t)), t \in[0, T)$, be a solution to the normalized Ricci flow equation (1) on a closed n-manifold $M$. Then $\lambda(g(t))$ increases whenever $\lambda(g(t)) \leq 0$, and the increasing is strict unless $g(t)$ is Einstein.

Proof. Consider the coupled equation

$$
\left\{\begin{array}{l}
\frac{\partial}{\partial t} g=-2 R i c+\frac{2 r}{n} g \\
\frac{\partial}{\partial t} f=-\Delta f+|\nabla f|^{2}-R+r .
\end{array}\right.
$$

Under this evolving equation, we have

$$
\frac{d}{d t} \mathcal{F}(g(t), f(t))=\int_{M}\left[2\left|R i c+\nabla^{2} f\right|^{2}-\frac{2}{n} r(R+\triangle f)\right] e^{-f} d v
$$


Let $\bar{f}$ be the eigenfunction of $-4 \triangle_{g(t)}+R(g(t))$. Denote by $\lambda(t)=\lambda(g(t))$, then

$$
\begin{aligned}
\frac{d}{d t} \lambda(t) & =\int_{M}\left[2\left|R i c+\nabla^{2} \bar{f}\right|^{2}-\frac{2}{n} r(R+\triangle \bar{f})\right] e^{-\bar{f}} d v \\
& \geq \int_{M}\left[\frac{2}{n}(R+\triangle \bar{f})^{2}-\frac{2}{n} r(R+\triangle \bar{f})\right] e^{-\bar{f}} d v \\
& \geq \frac{2}{n}\left(\int_{M}(R+\triangle \bar{f}) e^{-\bar{f}} d v\right)^{2}-\frac{2 r}{n} \int_{M}(R+\triangle \bar{f}) e^{-\bar{f}} d v \\
& =\frac{2}{n}\left(\int_{M}\left(R+|\nabla \bar{f}|^{2}\right) e^{-\bar{f}} d v\right)^{2}-\frac{2 r}{n} \int_{M}\left(R+|\nabla \bar{f}|^{2}\right) e^{-\bar{f}} d v \\
& =\frac{2}{n} \lambda(t)(\lambda(t)-r) .
\end{aligned}
$$

Now $\lambda(t) \leq r(t)$ and the monotonicity when $\lambda(t) \leq 0$ follows.

If $\frac{d}{d t} \lambda(t)=0$, then the equalities in the above estimate hold. So we have

$$
R+\triangle \bar{f}=\text { const. }
$$

and

$$
R i c+\nabla^{2} \bar{f}=\frac{1}{n}(R+\bar{f}) g .
$$

On the other hand, $e^{-\bar{f} / 2}$ is the only eigenfunction of $-4 \triangle+R$, so

$$
2 \triangle \bar{f}-|\nabla \bar{f}|^{2}+R=\lambda(t) .
$$

Thus $\triangle \bar{f}-|\nabla \bar{f}|^{2}=$ const., which equals zero since $\int_{M}\left(\triangle \bar{f}-|\nabla \bar{f}|^{2}\right) e^{-\bar{f}} d v=0$. By the maximal principle $f=$ const. over $M$. Hence $R=$ const. and Ric $=\frac{R}{n} g$. This proves the lemma.

Lemma 2.12 (Perelman). Let $(M, g(t)), t \in[0, T)$, be a solution to the Ricci flow equation (11) or (6)). If $\lambda(g(t))>0$ for all $t$, then $\nu(g(t))$ increases. Furthermore, the increasing is strict unless the solution is a shrinking Ricci soliton.

Proof. We only need to prove the unnormalized case, since the $\nu$ functional is invariant up to rescalings and diffeomorphism transformations, i.e., $\nu\left(\alpha \phi^{*} g\right)=\nu(g)$ for any constant $\alpha>0$ and diffeomorphism $\phi$ of $M$. Now for any times $t_{1}$ and $t_{2}$ such that $0 \leq t_{1}<t_{2}<T$, choose $\tau_{0}>0$ and $f_{0} \in C^{\infty}$ satisfying $\nu\left(g\left(t_{2}\right)\right)=\mu\left(g\left(t_{2}\right), \tau_{0}\right)=\mathcal{W}\left(g\left(t_{2}\right), f_{0}, \tau_{0}\right)$. Solving the equation [17, Equ. (3.3)]

$$
\left\{\begin{array}{l}
\frac{\partial}{\partial t} g=-2 \text { Ric } \\
\frac{\partial}{\partial t} f=-\triangle f+|\nabla f|^{2}-R+\frac{2 n}{\tau}, \\
\frac{\partial}{\partial t} \tau=-1 \\
f\left(t_{2}\right)=f_{0}, \tau\left(t_{2}\right)=\tau_{0},
\end{array}\right.
$$

and computing directly [14, under this evolving equation, we have

$$
\frac{d}{d t} \mathcal{W}=\int_{M} 2 \tau\left|R i c+\nabla^{2} f-\frac{1}{2 \tau} g\right|^{2}(4 \pi \tau)^{-n / 2} e^{-f} d v \geq 0
$$


Hence by the definitions of $\mu$ and $\nu$, we have

$$
\begin{aligned}
\nu\left(t_{1}\right) & \leq \mu\left(g\left(t_{1}\right), \tau_{0}+t_{2}-t_{1}\right) \\
& \leq \mathcal{W}\left(g\left(t_{1}\right), f\left(t_{1}\right), \tau_{0}+t_{2}-t_{1}\right) \\
& \leq \mathcal{W}\left(g\left(t_{2}\right), f\left(t_{2}\right), \tau_{0}\right)=\nu\left(t_{2}\right) .
\end{aligned}
$$

The equality doesn't hold unless the integrand equals zero, i.e., it is a shrinking Ricci soliton. On the other hand, $\nu$ remains obviously constant on a Ricci soliton by the invariance of this functional under changes by rescalings and diffeomorphism transformations.

\section{4-DIMENSIONAL NON-SINGULAR SOLUTIONS}

From now on we are concerned with Ricci flow on 4-manifolds. We will continue to use the same notations and conventions in $\S 2$. We assume all closed manifolds have constant volume 1 .

Lemma 3.1. Let $(M, g(t)), t \in[0, \infty)$ be a solution to (11) on a closed 4-manifold $M$. If $|R(g(t))| \leq C$ and $\breve{R}(g(t)) \leq-c<0$, where $C$ and $c$ are constants independent of $t$, then

$$
\int_{0}^{\infty} \int_{M}\left|\operatorname{Ric}^{o}(g(t))\right|^{2} d v d t<\infty .
$$

Proof. Note that, for $t \in[0, \infty)$,

$$
|R(g(t))|<C, \quad \text { and } \quad \breve{R}(g(t)) \leq-c<0,
$$

where $C$ and $c$ are constants independent of $t$. By Lemma 2.7, we have

$$
\int_{0}^{\infty} \int_{M}|R-r| d v d t<\infty .
$$

From the equation

we obtain

$$
\frac{\partial}{\partial t} R=\triangle R+2\left|R i c^{\mathrm{o}}\right|^{2}+\frac{2}{4} R(R-r)
$$

$$
\begin{aligned}
\int_{0}^{\infty} \int_{M} 2\left|R i c^{\circ}\right|^{2} d v d t & =\int_{0}^{\infty} \int_{M} \frac{\partial}{\partial t} R d v d t-\frac{1}{2} \int_{0}^{\infty} \int_{M} R(R-r) d v d t \\
& =\int_{0}^{\infty} \frac{\partial}{\partial t} r d t+\frac{1}{2} \int_{0}^{\infty} \int_{M} R(R-r) d v d t \\
& \leq \lim _{t \rightarrow \infty} \sup \left|r(g(t))-r_{0}\right|+\frac{C}{2} \int_{0}^{\infty} \int_{M}|R-r| d v d t \\
& \leq 2 C+\frac{C}{2} \int_{0}^{\infty} \int_{M}|R-r| d v d t<\infty
\end{aligned}
$$


Observe that $\breve{R}(g(t)) \leq \lambda_{M}=\bar{\lambda}_{M}$. Theorem 1.4 follows immediately from

Lemma 3.2. Let $M$ be a closed oriented 4-manifold $M$ and let $\{g(t)\}, t \in[0, \infty)$, be a solution to (11). If $|R(g(t))|<C$ and $\breve{R}(g(t)) \leq-c<0$, where $C$ and $c$ are constants independent of $t$, then

$$
2 \chi(M) \geq 3|\tau(M)|
$$

Furthermore, if $\bar{\lambda}_{M}<0$, then

$$
2 \chi(M)-3|\tau(M)| \geq \frac{1}{96 \pi^{2}} \bar{\lambda}_{M}^{2} .
$$

and any non-singular solution $\{g(t)\}, t \in[0, \infty)$ does not collapse.

Proof. From Lemma 3.1 we have

$$
\int_{m}^{m+1} \int_{M}\left|R i c^{\mathrm{o}}(g(t))\right|^{2} d v d t \longrightarrow 0
$$

when $m \longrightarrow \infty$.

By the Chern-Gauss-Bonnet formula and the Hirzebruch signature theorem, for any metric $g$ on $M$,

$$
\begin{gathered}
\chi(M)=\frac{1}{8 \pi^{2}} \int_{M}\left(\frac{R(g)^{2}}{24}+\left|W^{+}(g)\right|^{2}+\left|W^{-}(g)\right|^{2}-\frac{1}{2}\left|R i c^{\mathrm{o}}(g)\right|^{2}\right) d v, \quad \text { and } \\
\tau(M)=\frac{1}{12 \pi^{2}} \int_{M}\left(\left|W^{+}(g)\right|^{2}-\left|W^{-}(g)\right|^{2}\right) d v
\end{gathered}
$$

where $W^{+}(g)$ and $W^{-}(g)$ are the self-dual and anti-self-dual Weyl tensors respectively (cf. [1]). Thus

$$
\begin{aligned}
2 \chi(M)-3|\tau(M)| & \geq \liminf _{m \longrightarrow \infty} \frac{1}{4 \pi^{2}} \int_{m}^{m+1} \int_{M}\left(\frac{1}{24} R(g(t))^{2}-\frac{1}{2}\left|\operatorname{Ric}^{\mathrm{o}}(g(t))\right|^{2}\right) d v d t \\
& =\liminf _{m \longrightarrow \infty} \frac{1}{4 \pi^{2}} \int_{m}^{m+1} \int_{M} \frac{1}{24} R(g(t))^{2} d v d t \geq 0 .
\end{aligned}
$$

This proves the first inequality.

Observe that $\breve{R}(g(t)) \leq \lambda_{M}=\bar{\lambda}_{M}$. Note that

$$
\int_{m}^{m+1} \int_{M} R(g(t))^{2} d v d t \geq\left(\int_{m}^{m+1} r(g(t)) d t\right)^{2}=\left(\int_{m}^{m+1}(r-\breve{R}) d t+\int_{m}^{m+1} \breve{R} d t\right)^{2} .
$$

From Lemma 2.7, we have

$$
\int_{0}^{\infty}(r-\breve{R}) d t<\infty
$$

Thus

$$
\lim _{m \rightarrow \infty} \int_{m}^{m+1}(r-\breve{R}) d t=0
$$


as $r-\breve{R} \geq 0$. By taking $m \gg 1$ so that $\left|\int_{m}^{m+1}(r-\breve{R}) d t\right| \ll 1$, and

$$
\left(\int_{m}^{m+1}(r-\breve{R}) d t+\int_{m}^{m+1} \breve{R} d t\right)^{2} \geq\left(\int_{m}^{m+1}(r-\breve{R}) d t+\bar{\lambda}_{M}\right)^{2} .
$$

Thus we obtain

$$
2 \chi(M)-3|\tau(M)| \geq \liminf _{m \longrightarrow \infty} \frac{1}{96 \pi^{2}}\left(\int_{m}^{m+1}(r-\breve{R}) d t+\bar{\lambda}_{M}\right)^{2}=\frac{1}{96 \pi^{2}} \bar{\lambda}_{M}^{2} .
$$

The second inequality clearly implies that $\chi(M)>0$ whenever $\bar{\lambda}_{M}<0$. On the other hand, by Cheeger-Gromov's collapsing theorem (c.f. [2] [3]) it holds that $\chi(M)=0$ if $M$ collapse with bounded sectional curvature. This implies the desired result.

Proof of Theorem 1.2. By Theorem 1.1 and the Hitchin-Thorpe inequality for Einstein manifold it remains only to verify the inequality

$$
2 \chi(M) \geq 3|\tau(M)|
$$

in the case (1.1.5). By $\S 2$ we know that this only happens when $\breve{R}(g(t)) \leq-c<0$ and $\lambda(g(t)) \leq 0$, where $c$ is a constant independent of $t$. By Lemma 3.2 the desired result follows.

Proof of Corollary 1.3. If $M$ admits an $F$-structure of positive rank, then $\chi(M)=0$ (c.f. [2] [3]). If $M$ admits a shrinking soliton, by [8] the fundamental group $\pi_{1}(M)$ is finite, and so $b_{1}(M)=b_{3}(M)=0$ by the Poincarè duality. Hence $\chi(M) \geq 2$. By Theorem 1.2 the desired result follows.

Proof of Corollary [1.5. By Theorem 1 in [20], the Spin ${ }^{c}$ structure induced by a compatible almost complex structure on $(M, \omega)$ has Seiberg-Witten invariant equal to \pm 1 . Thus, by Corollary 4.4 in [15], we have

$$
\int_{M} R(g(t))^{2} d v \geq 32 \pi^{2}(2 \chi(M)+3 \tau(M)) .
$$

From the proof of Lemma 3.2, we know that

$$
\begin{aligned}
2 \chi(M)-3|\tau(M)| & \geq \liminf _{m \longrightarrow \infty} \frac{1}{4 \pi^{2}} \int_{m}^{m+1} \int_{M} \frac{1}{24} R(g(t))^{2} d v d t \\
& \geq \frac{1}{3}(2 \chi(M)+3 \tau(M)) .
\end{aligned}
$$

Hence

$$
\chi(M) \geq 3 \tau(M)
$$




\section{Proof of Theorem 1.6}

Let $(M, g(t)), t \in[0, \infty)$ be a non-singular solution to (11) on a closed oriented 4manifold $M$ with $\bar{\lambda}_{M}<0$. Since $|R m(g(t))|<C$, there is a constant $\varepsilon>0$ depending only on $C$ such that, for any $t, M_{t, \varepsilon}=\left\{x \in M: \operatorname{Vol}\left(B_{x}(1), g(t)\right)<\varepsilon\right\}$ admits an F-structure of positive rank, and the Euler number $\chi\left(M_{t, \varepsilon}\right)=0$ (cf. 3] 4] [1]). By Theorem 1.4, $\chi(M) \geq \frac{1}{96 \pi^{2}} \bar{\lambda}_{M}^{2}>0$. Hence, for any $t$, there is an $x \in M$ such that $\operatorname{Vol}\left(B_{x}(1), g(t)\right) \geq \varepsilon$.

By Lemma 2.7 and Lemma 3.1, we have

$$
\begin{gathered}
\int_{0}^{\infty} \int_{M} 2\left|R i c^{\mathrm{o}}\right|^{2} d v d t<\infty, \quad \int_{0}^{\infty}(r-\breve{R}) d t<\infty \quad \text { and } \\
\int_{0}^{\infty} \int_{M}|R-r| d v d t<\infty .
\end{gathered}
$$

Thus we may choose a sequence of times $\left\{t_{k}\right\}$ so that $t_{k} \longrightarrow \infty$ and

$$
\begin{gathered}
\nu(k)=\int_{M} 2\left|\operatorname{Ric}^{\mathrm{o}}\left(g\left(t_{k}\right)\right)\right|_{k}^{2} d v_{k} \longrightarrow 0, \quad\left|r\left(g\left(t_{k}\right)\right)-\breve{R}\left(g\left(t_{k}\right)\right)\right| \longrightarrow 0 \\
\mu(k)=\int_{M}\left|R\left(g\left(t_{k}\right)\right)-r\left(g\left(t_{k}\right)\right)\right| d v_{k} \longrightarrow 0
\end{gathered}
$$

as $k \longrightarrow \infty$.

We start the proof by proving three lemmas.

Lemma 4.1. There is a sequence points $\left\{x_{j, k} \in M\right\}, j=1, \cdots, m$, satisfying that, for any $j,\left(M, g\left(t_{k}\right), x_{j, k}\right) C^{\infty}$-converges to a complete Einstein manifold $\left(M_{j, \infty}, g_{j, \infty}, x_{j, \infty}\right)$, i.e. there are embeddings $F_{j, k, \rho}: B_{x_{j, \infty}}(\rho) \longrightarrow M$ such that, for any $\rho>0, F_{j, k, \rho}^{*} g\left(t_{k}\right)$ $C^{\infty}$-converges to $g_{j, \infty}$ on $B_{x_{j, \infty}}(\rho) \subset M_{j, \infty}$, and $F_{j, k, \rho}\left(x_{j, \infty}\right)=x_{j, k}$. Furthermore, all of the manifolds $M_{j, \infty}$ are distinct, and, for any $\rho>0$ and $k \gg 1,\left\{F_{j, k, \rho}\left(B_{x_{j, \infty}}(\rho)\right)\right\}$ are disjoint.

Proof. For each $g\left(t_{k}\right)$, choose a maximal number of disjoint unit balls $B_{x_{j, k}}(1) \subset\left(M, g\left(t_{k}\right)\right)$ so that $\operatorname{Vol}\left(B_{x_{j, k}}(1), g\left(t_{k}\right)\right) \geq \varepsilon$. Since $\operatorname{Vol}(M, g(t)) \equiv 1$, there is a uniform bound on the number of the balls. Therefore, by passing to a subsequence if necessary, we may assume that the maximal numbers of the disjoint unit balls for all $g\left(t_{k}\right)$ are the same, saying $\ell$. For every $1 \leq j \leq \ell$, by Hamilton's compactness theorem [1], $\left(M, g\left(t_{k}+t\right), x_{j, k}\right)$ $C^{\infty}$-converges to another normalized Ricci flow solution $\left(M_{j, \infty}, g_{j, \infty}(t), x_{j, \infty}\right)$ after passing to a subsequence. By Proposition [2.6. $\left(M_{j, \infty}, g_{j, \infty}(t), x_{j, \infty}\right)$ is a complete Einstein manifold with negative scalar curvature for every $t$. This proves that $\left(M, g\left(t_{k}\right), x_{j, k}\right)$ $C^{\infty}$-converges to a complete Einstein manifold $\left(M_{j, \infty}, g_{j, \infty}, x_{j, \infty}\right)$, where $g_{j, \infty} \equiv g_{j, \infty}(0)$, i.e. there are embeddings $F_{j, k, \rho}: B_{x_{j, \infty}}(\rho) \longrightarrow M$ such that, for any $\rho>0, F_{j, k, \rho}^{*} g\left(t_{k}\right)$ $C^{\infty}$-converges to $g_{j, \infty}$ on $B_{x_{j, \infty}}(\rho) \subset M_{j, \infty}$, and $F_{j, k, \rho}\left(x_{j, \infty}\right)=x_{j, k}$. Note that it is not necessary that all of the manifolds $M_{j, \infty}$ are distinct. Let $\left\{M_{j, \infty}\right\}, j=1, \cdots, m \leq \ell$, be 
the resulting collection of distinct manifolds. It is easy to see that, for any $\rho>0$ and $k \gg 1,\left\{F_{j, k, \rho}\left(B_{x_{j, \infty}}(\rho)\right)\right\}$ are disjoint, and $\sum \operatorname{Vol}\left(M_{j, \infty}, g_{j, \infty}\right) \leq 1$.

Remark: It is easy to see that two limit manifolds $M_{j_{1}, \infty}$ and $M_{j_{2}, \infty}$ are the same if and only if the distance $\operatorname{dist}_{g\left(t_{k}\right)}\left(x_{j_{1}, k}, x_{j_{2}, k}\right)$ is uniformly bounded above, independent of $k$.

By the above, $\left(M_{j, \infty}, g_{j, \infty}, x_{j, \infty}\right)$ satisfies $\left|R m\left(g_{j, \infty}\right)\right|<C$ and $\operatorname{Vol}\left(M_{j, \infty}, g_{j, \infty}\right) \leq 1$. By [5] there is a good chopping of $M_{j, \infty}$, i.e. an exhaustion, $\left\{U_{j, i}\right\}$, where every $U_{j, i}$ is a compact 4-submanifold with boundary $\partial U_{j, i}, \bigcup_{i=1}^{\infty} U_{j, i}=M_{j, \infty}$ such that

$$
\cdots \subset U_{j, i} \subset B_{x_{j, \infty}}(i) \subset U_{j, i+1} \subset B_{x_{j, \infty}}(i+1) \subset U_{j, i+2} \cdots \subset M_{j, \infty}
$$

satisfying that $\left|\operatorname{II}\left(\partial U_{j, i}\right)\right|<\Lambda$ for all $i$ and $\operatorname{Vol}\left(\partial U_{j, i},\left.g_{j, \infty}\right|_{\partial U_{j, i}}\right) \longrightarrow 0$ as $i \longrightarrow \infty$, where $\operatorname{II}\left(\partial U_{j, i}\right)$ is the second fundamental form of $\partial U_{j, i}$, and $\Lambda$ is a constant independent of $i$. By Lemma 4.1, we have

$$
\lim _{i \longrightarrow \infty}\left|\operatorname{Vol}\left(U_{j, i}, g_{j, \infty}\right)-\operatorname{Vol}\left(M_{j, \infty}, g_{j, \infty}\right)\right|=0, \quad \text { and } \quad \lim _{i \longrightarrow \infty} \operatorname{Vol}\left(\partial U_{j, i},\left.g_{j, \infty}\right|_{\partial U_{j, i}}\right)=0 .
$$

Lemma 4.2. Let $M_{k, i}=\coprod_{j=1}^{m} F_{j, k, i+1}\left(U_{j, i}\right) \subset\left(M, g\left(t_{k}\right)\right)$. Then $M_{k, i}$ is a 4-submanifold of $\left(M, g\left(t_{k}\right)\right)$ with boundary $\partial M_{k, i} \cong \coprod_{j=1}^{m} \partial U_{j, i}$, and every boundary component is a graph 3-manifolds for $i \gg 1$.

Proof. Since the second fundamental forms $\operatorname{II}\left(\partial U_{j, i}\right)$ of $\partial U_{j, i}$ have a uniform bound, the sectional curvatures of $\partial U_{j, i}$ have a uniform bound, i.e. $|\operatorname{Riem}|_{\partial \mathrm{U}_{\mathrm{j}, \mathrm{i}}}<\Lambda^{\prime}$. Because $\operatorname{dim} \partial \mathrm{U}_{\mathrm{j}, \mathrm{i}}=3$ and $\lim _{i \longrightarrow \infty} \operatorname{Vol}\left(\partial U_{j, i},\left.g_{j, \infty}\right|_{\partial U_{j, i}}\right)=0$, the components of $\partial U_{j, i}$ are graph 3-manifolds for $i \gg 1$ (cf. [5]). By Lemma 4.1, $F_{j, k, i+1}\left(U_{j, i}\right)$ are disjoint. Hence $M_{k, i}$ is a 4-submanifold of $M$ with boundary $\partial M_{k, i}=\coprod_{j=1}^{m} \partial U_{j, i}$. The desired result follows.

\section{Lemma 4.3.}

$$
\begin{gathered}
\lim _{k \longrightarrow \infty}\left|\sum_{j=1}^{m} \operatorname{Vol}\left(\mathrm{U}_{\mathrm{j}, \mathrm{i}}, \mathrm{F}_{\mathrm{j}, \mathrm{k}, \mathrm{i}+1}^{*} \mathrm{~g}\left(\mathrm{t}_{\mathrm{k}}\right)\right)-\operatorname{Vol}\left(\mathrm{M}, \mathrm{g}\left(\mathrm{t}_{\mathrm{k}}\right)\right)\right| \leq \overline{\mathrm{C}}\left(\sum_{\mathrm{j}=1}^{\mathrm{m}} \operatorname{Vol}\left(\partial \mathrm{U}_{\mathrm{j}, \mathrm{i}}, \mathrm{g}_{\mathrm{j}, \infty} \mid \partial \mathrm{U}_{\mathrm{j}, \mathrm{i}}\right)\right)^{\frac{1}{2}} \\
\text { and } \sum_{j=1}^{m} \operatorname{Vol}\left(\mathrm{M}_{\mathrm{j}, \infty}, \mathrm{g}_{\mathrm{j}, \infty}\right)=1
\end{gathered}
$$

where $\bar{C}$ is a constant independent of the indices.

Proof. We first claim that there is an $i_{0}>0$ such that, for any $i>i_{0}$, there is a $k_{0}$ satisfying that, for any $k>k_{0}, \operatorname{Vol}\left(\mathrm{B}_{\mathrm{y}}(1), \mathrm{g}\left(\mathrm{t}_{\mathrm{k}}\right)\right) \leq \varepsilon$ for all $y \in M-M_{k, i}$.

We may choose an $i_{0} \gg 1$ such that, for all $y_{\infty} \in \bigcup_{j=1}^{m}\left(M_{j, \infty}-B_{x_{j, \infty}}\left(i_{0}-2\right)\right)$, $\operatorname{Vol}\left(\mathrm{B}_{\mathrm{y}_{\infty}}(1), \mathrm{g}_{\mathrm{j}, \infty}\right) \leq \frac{1}{2} \varepsilon$. If the claim is false, for any fixed $i>i_{0}$, there is a subsequence of times $\left\{t_{k_{s}}\right\}$, and a sequence of points $\left\{y_{k_{s}}\right\}$ such that $y_{k_{s}} \in M-M_{k, i}$, and

$$
\operatorname{Vol}\left(\mathrm{B}_{\mathrm{y}_{\mathrm{s}}}(1), \mathrm{g}\left(\mathrm{t}_{\mathrm{k}_{\mathrm{s}}}\right)\right)>\varepsilon
$$


Observe that the distance $\operatorname{dist}_{\mathrm{g}\left(\mathrm{t}_{\mathrm{k}_{\mathrm{s}}}\right)}\left(\mathrm{y}_{\mathrm{k}_{\mathrm{s}}}, \mathrm{x}_{\mathrm{j}, \mathrm{k}_{\mathrm{s}}}\right) \longrightarrow \infty$ as $k_{s} \rightarrow \infty$ for all $1 \leq j \leq m$. Otherwise, assuming $\operatorname{dist}_{\mathrm{g}_{\left(\mathrm{t}_{\mathrm{s}}\right)}}\left(\mathrm{y}_{\mathrm{k}_{\mathrm{s}}}, \mathrm{x}_{\mathrm{j}, \mathrm{k}_{\mathrm{s}}}\right)<\rho$ for some $j$ and $\rho>0$, we get that $F_{j, k_{s}, \rho}^{-1}\left(y_{k_{s}}\right) \longrightarrow$ $y_{\infty} \in B_{x_{j, \infty}}(\rho)-B_{x_{j, \infty}}(i-1)$, and so

$$
\operatorname{Vol}\left(\mathrm{B}_{\mathrm{y}_{\mathrm{s}}}(1), \mathrm{g}\left(\mathrm{t}_{\mathrm{k}_{\mathrm{s}}}\right)\right) \longrightarrow \operatorname{Vol}\left(\mathrm{B}_{\mathrm{y}_{\infty}}(1), \mathrm{g}_{\mathrm{j}, \infty}\right) \leq \frac{1}{2} \varepsilon
$$

when $k_{s} \longrightarrow \infty$, since $F_{j, k_{s}, \rho}^{*} g\left(t_{k_{s}}\right) C^{\infty}$-converges to $g_{j, \infty}$. This contradicts to (11).

On the other hand, $\left(M, g\left(t_{k_{s}}\right), y_{k_{s}}\right) C^{\infty}$-converges to a complete Einstein manifold $\left(M_{\infty}, g_{\infty}, y_{\infty}\right)$, and $M_{\infty}$ is distinct from everyone of $M_{j, \infty}$ for $1 \leq j \leq \ell$ (cf. the remark after Lemma 4.1). This violates the choice of maximality of $m$. The claim follows.

By [5] and the above claim, for any $i>i_{0}$, there is a $k_{0}$ such that, for any $k>k_{0}$, $M-M_{k, i}$ admits an F-structure of positive rank, and $\chi\left(M-M_{k, i}\right)=0$. By ChernGauss-Bonnet theorem,

$$
\begin{aligned}
0=\chi\left(M-M_{k, i}\right)= & \frac{1}{8 \pi^{2}} \int_{M-M_{k, i}}\left(\frac{R\left(g\left(t_{k}\right)\right)^{2}}{24}+\left|W^{ \pm}\left(g\left(t_{k}\right)\right)\right|_{k}^{2}-\frac{1}{2}\left|\operatorname{Ric}^{\mathrm{o}}\left(g\left(t_{k}\right)\right)\right|_{k}^{2}\right) d v_{k} \\
& +\int_{\partial\left(M-M_{k, i}\right)} P_{k}(\mathrm{II}),
\end{aligned}
$$

where $P_{k}(\mathrm{II})$ is a polynomial of the second fundamental form $\mathrm{II}\left(\partial\left(M-M_{k, i}\right)\right)$ of $\partial(M-$ $\left.M_{k, i}\right)$ and its sectional curvature. Hence

$$
\int_{M-M_{k, i}} \frac{R\left(g\left(t_{k}\right)\right)^{2}}{24} d v_{k} \leq \int_{M} \frac{1}{2}\left|R i c^{\mathrm{o}}\left(g\left(t_{k}\right)\right)\right|_{k}^{2} d v_{k}-8 \pi^{2} \int_{\partial\left(M-M_{k, i}\right)} P_{k}(\mathrm{II}) .
$$

Since $F_{j, k, i+1}^{*} g\left(t_{k}\right) C^{\infty}$-converges to $g_{j, \infty}$ on $B_{x_{j, \infty}}(i+1) \subset M_{j, \infty}$, we have $\left|P_{k}(\mathrm{II})\right|<\bar{C}$, $k \gg 1$, where $\bar{C}$ is a constant depending only on the bounds $\Lambda$ of the second fundamental forms $\operatorname{II}\left(\partial U_{j, i}\right)$ of $\partial U_{j, i}$, and

$$
v(k):=\left|\operatorname{Vol}\left(\partial\left(\mathrm{M}-\mathrm{M}_{\mathrm{k}, \mathrm{i}}\right),\left.\mathrm{g}\left(\mathrm{t}_{\mathrm{k}}\right)\right|_{\partial\left(\mathrm{M}-\mathrm{M}_{\mathrm{k}, \mathrm{i}}\right)}\right)-\sum_{\mathrm{j}=1}^{\mathrm{m}} \operatorname{Vol}\left(\partial \mathrm{U}_{\mathrm{j}, \mathrm{i}},\left.\mathrm{g}_{\mathrm{j}, \infty}\right|_{\partial \mathrm{U}_{\mathrm{j}, \mathrm{i}}}\right)\right| \longrightarrow 0
$$

if $k \longrightarrow \infty$. Hence

$$
\int_{M-M_{k, i}} \frac{R\left(g\left(t_{k}\right)\right)^{2}}{24} d v_{k} \leq \frac{\nu(k)}{4}+\bar{C}\left(\sum_{j=1}^{m} \operatorname{Vol}\left(\partial \mathrm{U}_{\mathrm{j}, \mathrm{i}},\left.\mathrm{g}_{\mathrm{j}, \infty}\right|_{\partial \mathrm{U}_{\mathrm{j}, \mathrm{i}}}\right)+v(\mathrm{k})\right)
$$

where $\nu(k)$ is defined in equation (7).

Clearly,

$$
\begin{aligned}
& \left|r\left(g\left(t_{k}\right)\right)\right| \operatorname{Vol}\left(\mathrm{M}-\mathrm{M}_{\mathrm{k}, \mathrm{i}}, \mathrm{g}\left(\mathrm{t}_{\mathrm{k}}\right)\right)-\int_{\mathrm{M}-\mathrm{M}_{\mathrm{k}, \mathrm{i}}}\left|\mathrm{R}\left(\mathrm{g}\left(\mathrm{t}_{\mathrm{k}}\right)\right)\right| \mathrm{dv}_{\mathrm{k}} \\
\leq & \int_{M-M_{k, i}}\left|R\left(g\left(t_{k}\right)\right)-r\left(g\left(t_{k}\right)\right)\right| d v_{k} \leq \mu(k),
\end{aligned}
$$

where $\mu(k)$ is as in (8). 


$$
\begin{aligned}
\int_{M-M_{k, i}}\left|R\left(g\left(t_{k}\right)\right)\right| d v_{k} & \leq\left(\int_{M-M_{k, i}} R\left(g\left(t_{k}\right)\right)^{2} d v_{k}\right)^{\frac{1}{2}} \operatorname{Vol}\left(\mathrm{M}-\mathrm{M}_{\mathrm{k}, \mathrm{i}}, \mathrm{g}\left(\mathrm{t}_{\mathrm{k}}\right)\right)^{\frac{1}{2}} \\
& \leq\left(\int_{M-M_{k, i}} R\left(g\left(t_{k}\right)\right)^{2} d v_{k}\right)^{\frac{1}{2}} .
\end{aligned}
$$

Thus

$$
\begin{aligned}
\operatorname{Vol}\left(\mathrm{M}-\mathrm{M}_{\mathrm{k}, \mathrm{i}}, \mathrm{g}\left(\mathrm{t}_{\mathrm{k}}\right)\right) \leq & \frac{2}{\left|r_{\infty}\right|}(\mu(k)+(6 \nu(k) \\
& \left.\left.+\bar{C}\left(\sum_{j=1}^{m} \operatorname{Vol}\left(\partial \mathrm{U}_{\mathrm{j}, \mathrm{i}},\left.\mathrm{g}_{\mathrm{j}, \infty}\right|_{\partial \mathrm{U}_{\mathrm{j}, \mathrm{i}}}\right)+v(\mathrm{k})\right)\right)^{\frac{1}{2}}\right),
\end{aligned}
$$

where we used the fact that $r\left(g\left(t_{k}\right)\right) \longrightarrow r_{\infty}<0$ if $k \longrightarrow \infty$ (cf. the proof of Proposition 2.6.) Therefore,

$$
\begin{aligned}
\left|\sum_{j=1}^{m} \operatorname{Vol}\left(\mathrm{U}_{\mathrm{j}, \mathrm{i}}, \mathrm{g}_{\mathrm{j}, \infty}\right)-1\right| & =\lim _{k \longrightarrow \infty}\left|\operatorname{Vol}\left(\mathrm{M}_{\mathrm{k}, \mathrm{i}}, \mathrm{g}\left(\mathrm{t}_{\mathrm{k}}\right)\right)-1\right| \\
& \leq \bar{C}\left(\sum_{j=1}^{m} \operatorname{Vol}\left(\partial \mathrm{U}_{\mathrm{j}, \mathrm{i}},\left.\mathrm{g}_{\mathrm{j}, \infty}\right|_{\partial \mathrm{U}_{\mathrm{j}, \mathrm{i}}}\right)\right)^{\frac{1}{2}} .
\end{aligned}
$$

By letting $i \longrightarrow \infty$, we get the second equality in the lemma.

Proof of Theorem 1.6. By Lemma 4.3, for any $\delta>0$, we can choose $i \gg 1$ and $k \gg 1$ such that $\operatorname{Vol}\left(\mathrm{M}-\mathrm{M}_{\mathrm{k}, \mathrm{i}}, \mathrm{g}\left(\mathrm{t}_{\mathrm{k}}\right)\right)<\delta$. Let $T=t_{k}$ and $M^{\varepsilon}=M_{k, i}$. The desired results follows by Lemmas 4.1, 4.2 and 4.3 .

\section{PROOF OF TheOREM 1.7}

Let us first recall some facts about Seiberg-Witten equations, which will be used to prove Theorem 1.7 (See [15] for details). Let $(M, g)$ be a compact oriented Riemannian 4-manifold with a $\operatorname{Spin}^{\mathrm{c}}$ structure $\mathfrak{c}$. Let $b_{2}^{+}(M)$ denote the dimension of the space of self-dual harmonic 2-forms in $M$. Let $S_{\mathfrak{c}}^{ \pm}$denote the $\operatorname{Spin}^{\mathrm{c}}$-bundles associated to $\mathfrak{c}$, and let $L$ be the determinant line bundle of $\mathfrak{c}$. There is a well-defined Dirac operator

$$
\mathcal{D}_{A}: \Gamma\left(S_{\mathfrak{c}}^{+}\right) \longrightarrow \Gamma\left(S_{\mathfrak{c}}^{-}\right)
$$

Let $c: \wedge^{*} T^{*} M \longrightarrow \operatorname{End}\left(S_{\mathfrak{c}}^{+} \oplus S_{\mathfrak{c}}^{-}\right)$denote the Clifford multiplication on the $\mathrm{Spin}^{\mathrm{c}}$ bundles, and, for any $\phi \in \Gamma\left(S^{ \pm}\right)$, let

$$
q(\phi)=\bar{\phi} \otimes \phi-\frac{1}{2}|\phi|^{2} \mathrm{id} .
$$


The Seiberg-Witten equations read

$$
\begin{gathered}
\mathcal{D}_{A} \phi=0 \\
c\left(F_{A}^{+}\right)=q(\phi)
\end{gathered}
$$

where the unknowns are a hermitian connection $A$ on $L$ and a section $\phi \in \Gamma\left(S_{\mathfrak{c}}^{+}\right)$, and $F_{A}^{+}$ is the self-dual part of the curvature of $A$. A resolution of the Seiberg-Witten equations is called reducible if $\phi \equiv 0$; otherwise, it is called irreducible.

Let $(M, g(t)), t \in[0, \infty)$ be a non-singular solution to (11) on a closed oriented 4manifold $M$ with $\bar{\lambda}_{M}<0$. We will continue to assume the volume of $(M, g(t))$ is 1 . Let $\breve{R}(g(t))$ denote the minimum of the scalar curvature of $g(t)$. Recall that $\breve{R}(g(t)) \leq \bar{\lambda}_{M}$. Assume that $M$ admits a symplectic structure $\omega$ satisfying that $b_{2}^{+}(M)>1$ and $\chi(M)=$ $3 \tau(M)$. Let $t_{k}, x_{j, k}, U_{j, i}, M_{j, \infty}$ and $F_{j, k, \rho}$ be the same as in Section 4 . By Theorem 1 in 20], the $\operatorname{Spin}^{c}$ structure induced by a compatible almost complex structure $J$ on $(M, \omega)$ has Seiberg-Witten invariant equal to \pm 1 . Hence, for any $k$, there is an irreducible solution $\left(\phi_{k}, A_{k}\right)$ to the Seiberg-Witten equations (cf. [15]). For the sake of simplicity we will use $|\cdot|_{k}$ to denote the norm with respect to the metric $g\left(t_{k}\right)$.

\section{Lemma 5.1.}

$$
\begin{gathered}
8 \int_{M}\left|F_{A_{k}}^{+}\right|_{k}^{2} d v_{k} \geq \int_{M} R\left(g\left(t_{k}\right)\right)^{2} d v_{k}-\int_{M} 48 \pi^{2}\left|\operatorname{Ric}^{o}\left(g\left(t_{k}\right)\right)\right|_{k}^{2} d v_{k}, \\
\text { and } \lim _{k \longrightarrow \infty} \int_{M}\left|\nabla^{k} F_{A_{k}}^{+}\right|_{k}^{2} d v_{k}=0 .
\end{gathered}
$$

Proof. The Bochner formula implies that

$$
\begin{aligned}
& 0=\frac{1}{2} \Delta_{k}\left|\phi_{k}\right|_{k}^{2}+\left|\nabla^{A_{k}} \phi_{k}\right|_{k}^{2}+\frac{R\left(g\left(t_{k}\right)\right)}{4}\left|\phi_{k}\right|_{k}^{2}+\frac{1}{4}\left|\phi_{k}\right|_{k}^{4}, \\
& 4 \int_{M}\left|\nabla^{A_{k}} \phi_{k}\right|_{k}^{2} d v_{k}=-\int_{M}\left(R\left(g\left(t_{k}\right)\right)\left|\phi_{k}\right|_{k}^{2}+\left|\phi_{k}\right|_{k}^{4}\right) d v_{k} .
\end{aligned}
$$

From the estimate $\left|\phi_{k}\right|_{k}^{2} \leq-\breve{R}\left(g\left(t_{k}\right)\right)$ (cf. [15]) and $-R\left(g\left(t_{k}\right)\right) \leq-\breve{R}\left(g\left(t_{k}\right)\right)<0$ we get that

$$
4 \int_{M}\left|\nabla^{A_{k}} \phi_{k}\right|_{k}^{2} d v_{k} \leq \int_{M} \breve{R}\left(g\left(t_{k}\right)\right)^{2} d v_{k}-\int_{M}\left|\phi_{k}\right|_{k}^{4} d v_{k} .
$$

By the second equation of the Seiberg-Witten equations and $\chi(M)=3 \tau(M)$,

$$
\begin{aligned}
\int_{M}\left|\phi_{k}\right|_{k}^{4} d v_{k} & =8 \int_{M}\left|F_{A_{k}}^{+}\right|_{k}^{2} d v_{k} \\
& \geq 32 \pi^{2}\left[c_{1}^{+}\right]^{2}[M] \geq 32 \pi^{2}\left[c_{1}\right]^{2}[M] \\
& =32 \pi^{2}(2 \chi(M)+3 \tau(M)) \\
& =96 \pi^{2}(2 \chi(M)-3 \tau(M)) \\
& \geq \int_{M} R\left(g\left(t_{k}\right)\right)^{2} d v_{k}-\int_{M} 48 \pi^{2}\left|\operatorname{Ric}^{\mathrm{o}}\left(g\left(t_{k}\right)\right)\right|_{k}^{2} d v_{k}
\end{aligned}
$$


where the last inequality follows by the Chern-Gauss-Bonnet formula and Hirzebruch's signature formula (cf. section 4). Thus

$$
8 \int_{M}\left|F_{A_{k}}^{+}\right|_{k}^{2} d v_{k} \geq \int_{M} R\left(g\left(t_{k}\right)\right)^{2} d v_{k}-\int_{M} 48 \pi^{2}\left|\operatorname{Ric}^{\mathrm{o}}\left(g\left(t_{k}\right)\right)\right|_{k}^{2} d v_{k},
$$

where $c_{1}^{+}$is the self-dual part of the harmonic form representing the first Chern class $c_{1}$ of $M$. Hence, by (7) (8), we have

$$
\begin{aligned}
4 \int_{M}\left|\nabla^{A_{k}} \phi_{k}\right|_{k}^{2} d v_{k} & \leq \int_{M}\left(\breve{R}\left(g\left(t_{k}\right)\right)^{2}-R\left(g\left(t_{k}\right)\right)^{2}\right) d v_{k}+\int_{M} 48 \pi^{2}\left|\operatorname{Ric}^{\mathrm{o}}\left(g\left(t_{k}\right)\right)\right|_{k}^{2} d v_{k} \\
& \leq C\left|\breve{R}\left(g\left(t_{k}\right)\right)-r\left(g\left(t_{k}\right)\right)\right|+\int_{M} 48 \pi^{2}\left|\operatorname{Ric}^{\mathrm{o}}\left(g\left(t_{k}\right)\right)\right|_{k}^{2} d v_{k} \longrightarrow 0
\end{aligned}
$$

$k \longrightarrow 0$, where $C$ is a constant independent of $k$. By the second one of the SeibergWitten equations again (cf. [15]),

$$
\left|\nabla^{k} F_{A_{k}}^{+}\right|_{k}^{2} \leq \frac{1}{2}\left|\phi_{k}\right|_{k}^{2}\left|\nabla^{A_{k}} \phi_{k}\right|_{k}^{2}
$$

where $\nabla^{k}$ is the connection induced by Levi-civita connection. Hence

$$
\int_{M}\left|\nabla^{k} F_{A_{k}}^{+}\right|_{k}^{2} d v_{k} \leq \frac{1}{2}\left|\breve{R}\left(g\left(t_{k}\right)\right)\right| \int_{M}\left|\nabla^{A_{k}} \phi_{k}\right|_{k}^{2} d v_{k} \longrightarrow 0
$$

when $k \longrightarrow \infty$.

Regard $F_{A_{k}}^{+}$as self-dual 2-forms of $g^{\prime}\left(t_{k}\right)$ on $U_{j, i} \subset M_{j, \infty}$, where $g^{\prime}\left(t_{k}\right)=F_{j, k, i+1}^{*} g\left(t_{k}\right)$. Since $\left|F_{A_{k}}^{+}\right|_{k}^{2}=\frac{1}{8}\left|\phi_{k}\right|_{k}^{4} \leq \frac{1}{8} \breve{R}\left(g\left(t_{k}\right)\right)^{2} \leq C$, where $C$ is a constant independent of $k$, $F_{A_{k}}^{+} \in L_{1}^{2}\left(g^{\prime}\left(t_{k}\right)\right)$, and

$$
\left\|F_{A_{k}}^{+}\right\|_{L_{1}^{2}\left(g^{\prime}\left(t_{k}\right)\right)} \leq C^{\prime}
$$

where $C^{\prime}$ is a constant independent of $k$. Note that $\|\cdot\|_{L_{1}^{2}\left(g_{j, \infty}\right)} \leq 2\|\cdot\|_{L_{1}^{2}\left(g^{\prime}\left(t_{k}\right)\right)}$ for $k \gg 1$ since $g^{\prime}\left(t_{k}\right) C^{\infty}$-converges to $g_{j, \infty}$ on $U_{j, i}$. Thus, by passing to a subsequence, $F_{A_{k}}^{+}$ $L_{1}^{2}$-converges to a 2 -form $\Omega_{j} \in L_{1}^{2}\left(g_{j, \infty}\right)$, which is a self-dual 2 -form of $g_{j, \infty}$.

Lemma 5.2. For any $j, \Omega_{j}$ is a smooth self-dual 2-form on $U_{j, i}-\partial U_{j, i}$ such that $\nabla^{\infty} \Omega_{j} \equiv 0$, and $\left|\Omega_{j}\right|_{\infty} \equiv$ cont. $\neq 0$, where $\nabla^{\infty}$ is the connection induced by the Levicivita connection of $g_{j, \infty}$.

Proof. Note that

$$
0 \leq \int_{U_{j, i}}\left|\nabla^{\infty} \Omega_{j}\right|_{\infty}^{2} d v_{\infty}=\lim _{k \longrightarrow \infty} \int_{U_{j, i}}\left|\nabla^{\infty} F_{A_{k}}^{+}\right|_{\infty}^{2} d v_{\infty} \leq \lim _{k \longrightarrow \infty} \int_{M}\left|\nabla^{k} F_{A_{k}}^{+}\right|_{k}^{2} d v_{k}=0 .
$$

It is easy to see that $\Omega_{j}$ is a weak solution of the elliptic equation $\left(d+d^{*}\right) \Omega_{j}=0$ on $U_{j, i}$. By elliptic equation theory, $\Omega_{j}$ is a smooth self-dual 2-form on $U_{j, i}-\partial U_{j, i}$, and $\nabla^{\infty} \Omega_{j} \equiv 0$. 
Now we claim that, for any $j$ and $i \gg 1, \int_{U_{j, i}}\left|\Omega_{j}\right|_{\infty}^{2} d v_{\infty} \neq 0$. If it is not true, there is a $j_{1}$ such that $\int_{U_{j_{1}, i}}\left|\Omega_{j_{1}}\right|_{\infty}^{2} d v_{\infty} \equiv 0$. Note that, by the results in Section 4 ,

$$
\begin{aligned}
\int_{U_{j, i}}\left|\Omega_{j}\right|_{\infty}^{2} d v_{\infty}=\lim _{k \rightarrow \infty} \int_{U_{j, i}}\left|F_{A_{k}}^{+}\right|_{\infty}^{2} d v_{\infty} & =\lim _{k \rightarrow \infty} \int_{U_{j, i}}\left|F_{A_{k}}^{+}\right|_{k}^{2} d v_{k} \\
& \leq \frac{1}{8} \lim _{k \longrightarrow \infty} \breve{R}\left(g\left(t_{k}\right)\right)^{2} \operatorname{Vol}\left(\mathrm{U}_{\mathrm{j}, \mathrm{i}}, \mathrm{g}^{\prime}\left(\mathrm{t}_{\mathrm{k}}\right)\right) \\
& =\frac{1}{8} r_{\infty}^{2} \operatorname{Vol}\left(\mathrm{U}_{\mathrm{j}, \mathrm{i}}, \mathrm{g}_{\mathrm{j}, \infty}\right) \\
\left|\int_{M}\left(R\left(g\left(t_{k}\right)\right)^{2}-r_{\infty}^{2}\right) d v_{k}\right| \leq C \int_{M}\left(\mid R\left(g\left(t_{k}\right)\right.\right. & \left.-r\left(g\left(t_{k}\right)\right)|+| r_{\infty}-r\left(g\left(t_{k}\right)\right) \mid\right) d v_{k} \longrightarrow 0
\end{aligned}
$$

and

$$
\begin{aligned}
\left.\lim _{k \rightarrow \infty}\left|\int_{M}\right| F_{A_{k}}^{+}\right|_{k} ^{2} d v_{k}-\sum_{j=1}^{m} \int_{U_{j i}}\left|F_{A_{k}}^{+}\right|_{k}^{2} d v_{k} \mid= & \lim _{k \rightarrow \infty} \int_{M-M_{k, i}}\left|F_{A_{k}}^{+}\right|_{k}^{2} d v_{k} \\
\leq & C \lim _{k \rightarrow \infty} \mid \sum_{j=1}^{m} \operatorname{Vol}\left(\mathrm{U}_{\mathrm{j}, \mathrm{i}}, \mathrm{g}^{\prime}\left(\mathrm{t}_{\mathrm{k}}\right)\right) \\
& -\operatorname{Vol}\left(\mathrm{M}, \mathrm{g}\left(\mathrm{t}_{\mathrm{k}}\right)\right) \mid \\
\leq & \bar{C}\left(\sum_{j=1}^{m} \operatorname{Vol}\left(\partial \mathrm{U}_{\mathrm{j}, \mathrm{i}},\left.\mathrm{g}_{\mathrm{j}, \infty}\right|_{\partial \mathrm{U}_{\mathrm{j}, \mathrm{i}}}\right)\right)^{\frac{1}{2}}
\end{aligned}
$$

if $k \longrightarrow \infty$, where $C$ and $\bar{C}$ are constants independent of $k$. By Lemma 5.1, we obtain

$$
\begin{aligned}
r_{\infty}^{2} \sum_{j \neq j_{1}} \operatorname{Vol}\left(\mathrm{U}_{\mathrm{j}, \mathrm{i}}, \mathrm{g}_{\mathrm{j}, \infty}\right) & \geq \sum_{j=1}^{m} \int_{U_{j, i}} 8\left|\Omega_{j}\right|_{\infty}^{2} d v_{\infty}=\lim _{k \rightarrow \infty} \int_{M_{k, i}} 8\left|F_{A_{k}}^{+}\right|_{k}^{2} d v_{k} \\
& \geq \lim _{k \rightarrow \infty} \int_{M} 8\left|F_{A_{k}}^{+}\right|_{k}^{2} d v_{k}-\bar{C}\left(\sum_{j=1}^{m} \operatorname{Vol}\left(\partial \mathrm{U}_{\mathrm{j}, \mathrm{i}},\left.\mathrm{g}_{\mathrm{j}, \infty}\right|_{\partial \mathrm{U}_{\mathrm{j}, \mathrm{i}}}\right)\right)^{\frac{1}{2}} \\
& \geq \lim _{k \longrightarrow \infty} \int_{M}\left(R\left(g\left(t_{k}\right)\right)^{2} d v_{k}-\bar{C}\left(\sum_{j=1}^{m} \operatorname{Vol}\left(\partial \mathrm{U}_{\mathrm{j}, \mathrm{i}},\left.\mathrm{g}_{\mathrm{j}, \infty}\right|_{\partial \mathrm{U}_{\mathrm{j}, \mathrm{i}}}\right)\right)^{\frac{1}{2}}\right. \\
& =r_{\infty}^{2}-\bar{C}\left(\sum_{j=1}^{m} \operatorname{Vol}\left(\partial \mathrm{U}_{\mathrm{j}, \mathrm{i}},\left.\mathrm{g}_{\mathrm{j}, \infty}\right|_{\partial \mathrm{U}_{\mathrm{j}, \mathrm{i}}}\right)\right)^{\frac{1}{2}} .
\end{aligned}
$$

Note that, for $i \gg 1$,

$$
1 \gg \bar{C}\left(\sum_{j=1}^{m} \operatorname{Vol}\left(\partial \mathrm{U}_{\mathrm{j}, \mathrm{i}},\left.\mathrm{g}_{\mathrm{j}, \infty}\right|_{\partial \mathrm{U}_{\mathrm{j}, \mathrm{i}}}\right)\right)^{\frac{1}{2}} \geq \mathrm{r}_{\infty}^{2} \operatorname{Vol}\left(\mathrm{U}_{\mathrm{j}_{1}, \mathrm{i}}, \mathrm{g}_{\mathrm{j} 1, \infty}\right)
$$

A contradiction. Thus, for any $j, \int_{U_{j, i}}\left|\Omega_{j}\right|_{\infty}^{2} d v_{\infty} \neq 0$. Thus we obtain that $\nabla^{\infty} \Omega_{j} \equiv 0$, $\left|\Omega_{j}\right|_{\infty} \equiv$ cont.$\neq 0$. 
Proof of Theorem 1.7. Since all $\Omega_{j}, 1 \leq j \leq m$, are self-dual 2-forms, and $\nabla^{\infty} \Omega_{j} \equiv 0$, $\left|\Omega_{j}\right|_{\infty} \equiv$ cont. $\neq 0$. Hence, on any $U_{j, i}, g_{j, \infty}$ is a Kähler metric with Kähler form $\sqrt{2} \frac{\Omega_{j}}{\left|\Omega_{j}\right|}$. By Lemma 4.1, $g_{j, \infty}$ is a Kähler-Einstein metric on $M_{j, \infty}$.

Now, by the Chern-Gauss-Bonett theorem and the Hirzebruch theorem,

$$
\begin{aligned}
0=\chi(M)-3 \tau(M) & \geq \liminf _{k \rightarrow \infty} \frac{1}{2 \pi^{2}}\left(\int_{M}\left|W^{-}\left(g\left(t_{k}\right)\right)\right|_{k}^{2} d v_{k}-\frac{1}{4} \int_{M}\left|\operatorname{Ric}^{\mathrm{o}}\left(g\left(t_{k}\right)\right)\right|_{k}^{2} d v_{k}\right) \\
& =\liminf _{k \rightarrow \infty} \frac{1}{2 \pi^{2}} \int_{M}\left|W^{-}\left(g\left(t_{k}\right)\right)\right|_{k}^{2} d v_{k} \geq 0,
\end{aligned}
$$

where $W^{-}$is the anti-self-dual Weyl tensor. Thus

$$
\liminf _{k \rightarrow \infty} \int_{M}\left|W^{-}\left(g\left(t_{k}\right)\right)\right|_{k}^{2} d v_{k}=0
$$

Therefore, for any $j$,

$$
0 \leq \int_{M_{j, \infty}}\left|W^{-}\left(g_{j, \infty}\right)\right|_{\infty}^{2} d v_{\infty} \leq \liminf _{k \longrightarrow \infty} \int_{M}\left|W^{-}\left(g\left(t_{k}\right)\right)\right|_{k}^{2} d v_{k}=0
$$

Hence $g_{j, \infty}$ is a Kähler-Einstein metric with $W^{-}\left(g_{j, \infty}\right) \equiv 0$. This implies that $g_{j, \infty}$ is a complex hyperbolic metric by the proof of Theorem 4.5 in [15. The desired result follows.

\section{REFERENCES}

[1] M. T. Anderson, Degeneration of metrics with bounded curvature and applications to critical metrics of Riemannian functionals, Proceeding of Sympoia in Pure Mathematics, 54 (1993), 53-79.

[2] A. L. Besse, Einstein manifolds, Ergebnisse der Math. Springer-Verlag, Berlin-New York 1987.

[3] J. Cheeger and M. Gromov, Collapsing Riemannian Manifolds while keeping their curvature bounded I, J.Diff.Geom. 23, (1986), 309-364.

[4] J. Cheeger and M. Gromov, Collapsing Riemannian Manifolds while keeping their curvature bounded II, J.Diff.Geom. 32, (1990), 269-298.

[5] J.Cheeger, M.Gromov, On the characteristic numbers of complete manifolds of bounded curvature and finite volume, H.E.Rauch Memorial Volume I, Springer, Berlin, 1985, 115-154.

[6] J. Cheeger, M. Gromov and M. Taylor, Finite propagation speed, kernal estimates for functions of the Laplace operatro, and the geometry of complete Riemannian manifolds, J. Diff. Geom. 17 (1982), 15-53.

[7] H.D. Cao and X.P. Zhu, A complete proof of the Poincaré and geometrization conjecturesapplication of the Hamilton-Perelman theory of the Ricci flow, Asian J. Math. 10 (2006) 165-492.

[8] F. Fang and Y.G. Zhang., Perelman's $\lambda$-functional and the Seiberg-Witten equations, preprint 2006

[9] M. Fernández-López and E. García-Río, A remark on compact Ricci solitons, preprint.

[10] R. Hamilton, Three-manifolds with positive Ricci curvature, J. Diff. Geom. 17 (1982) 255-306.

[11] R. Hamilton, A compactness property for solutions of the Ricci flow, Amer. J. Math. 117 (1995) 545-574.

[12] R. Hamilton, Non-singular solutions of the Ricci flow on three-manifolds, Comm. Anal. and Geom. 7 (1999) 695-729.

[13] T. Ivey, Ricci solitons on compact three-manifolds, Diff. Geom. Appl. 3 (1993) 301-307.

[14] B. Kleiner and J. Lott, Notes on Perelman's Papers, arXiv:math.DG/0605667.

[15] C. LeBrun, Four-Dimensional Einstein Manifolds and Beyond, in Surveys in Differential Geometry, vol VI: Essays on Einstein Manifolds, 247-285. 
[16] M. Feldman, T. Ilmanen and L. Ni, Entropy and reduced distance for Ricci expanders, J. Geom. Anal. 15 (2005).

[17] G. Perelman, The entropy formula for the Ricci flow and its geometric applications, arXiv:math.DG/0211159

[18] G. Perelman, Ricci flow with surgery on three-manifolds, arXiv:math.DG/0303109

[19] O. S. Rothaus, Logarithmic Sobolev inequalities and the spectrum of Schrödinger operators, J. Func. Anal. 42 (1981) 110-120.

[20] C. H. Taubes, More constraints on symplectic forms from Seiberg-Witten invariants, Math. Res. Lett. 2 (1995), 9-13.

[21] R.Schoen and S. T. Yau, Lectures on differential geometry, in Conference Proceedings and Lecture Notes in Geometry and Topology, 1, International Press Publications, 1994.

Nankai Institute of Mathematics, Weijin Road 94, Tianjin 300071, P.R.China

Department of Mathematics, Capital Normal University, Beijing, P.R.China

E-mail address: ffang@nankai.edu.cn

Department of Mathematics, Capital Normal University, Beijing, P.R.China

Nankai Institute of Mathematics, Weijin Road 94, Tianjin 300071, P.R.China 